\title{
Vertrauenswürdige Kommunikation: Ergebnisse einer Umfrage
}

\author{
Andrea Herrmann ${ }^{1}$ \\ Online publiziert: 20. Mai 2020 \\ (c) Der/die Autor(en) 2020
}

\section{Zusammenfassung}

Datenschutz ist Vertrauenssache: Ein Kunde vertraut dem Anbieter einer digitalen Dienstleistung personenbezogene und persönliche Daten an und hofft, dass dieser sie erfolgreich beschützt und der Versuchung widersteht, sie zu unlauteren Zwecken zu missbrauchen. Dieses Vertrauen wird immer wieder enttäuscht. Sammelt eine Firma zentral große Mengen personenbezogener Daten ihrer Kunden, so bilden diese den Schatz der Firma, der ggf. verkauft oder gestohlen werden kann.

Der einzige sichere Schutz gegen solche Versuchungen besteht darin, eine zentrale Datenansammlung zu vermeiden. Technisch gibt es die Möglichkeit dazu, indem man für Kommunikation und Datenhaltung dezentrale, vertrauenswürdige Peer-to-Peer-Software (P2P) verwendet.

Im GI-Radar 249 hat die Gesellschaft für Informatik im Oktober 2019 eine Umfrage zu verschiedenen solchen Technologien durchgeführt. Herzlichen Dank den 148 Teilnehmer*innen, die den Fragebogen vollständig ausgefüllt haben!

In diesem Artikel werden zunächst drei Initiativen, dann einige Technologien und zuletzt die Ergebnisse der Umfrage vorgestellt.

\section{Initiativen für ein dezentrales Internet}

Obwohl das Internet von Anfang an dezentral konzipiert war, gerät dies immer wieder in Vergessenheit. Drei Initiativen rufen uns dieses Prinzip des WWW wieder in Erinnerung.

Die Declaration of Digital Independence Larry Sanger, Mitbegründer von Wikipedia, hat eine Erklärung der digitalen Unabhängigkeit formuliert, die das Recht des Individuums an seinen Daten betont. Da die Unternehmen, die soziale Netzwerke zur Verfügung stellen, bisher nicht sorgfältig genug mit diesen Daten umgegangen sind, ruft Sanger dazu auf, neue, dezentralisierte soziale Netzwerke zu entwickeln, Unternehmen entsprechend ihrem Datenschutz zu beurteilen und unzureichende Netzwerke zu verlassen. Ziel ist ein dezentralisiertes Netzwerk unabhängiger Individuen. Auf seiner Webseite kann man diese Erklärung unterzeichnen $[1]$.

Manifest „Holen wir uns das Web zurück!“ Eine ähnliche Initiative gibt es aus Deutschland von Kai Bösefeldt (auch in

Andrea Herrmann

andrea.herrmann@fh-dortmund.de

1 Fachhochschule Dortmund, Dortmund, Deutschland englischer Übersetzung verfügbar). Es geht um den Schutz des Internets, inzwischen die virtuelle Heimat vieler Menschen. Das Internet der Zukunft soll dezentral ausgelegt sein, resistent gegen Zentralismus, Zwang und Zensur. Die technische Grundlage dafür sind vertrauenswürdige Kommunikationstechnologien, die noch zu entwickeln sind. Anforderungen an eine solche Technologie sind ebenfalls Teil des Manifests [2].

Die Indie-Web-Bewegung Das Internet als dezentrales Netzwerk, dessen Knoten Menschen sind, soll durch passende Technologien wieder aufleben. Jeder besitzt dort seinen eigenen Raum, seine eigene Webseite, seine eigenen Daten [3].

\section{Dezentrale Technologien}

Die aktuell beliebtesten Online-Dienstleister wie Google, Facebook und Twitter erfüllen wichtige Bedürfnisse ihrer Kunden nach Information und Kommunikation. Darum ist es kein Zufall, dass gerade solche erfolgreich verbreiteten, jedoch kostenlosen Anwendungen oft den Geschäftszweck verfolgen, Kundendaten zu sammeln. Im Folgenden werden einige Alternativen zu den zentralisierten Marktführern vorgestellt. 
Tab. 1 Antworten auf die Frage „Würden Sie diese Lösung empfehlen?“

\begin{tabular}{lllllll}
\hline Lösung & $\begin{array}{l}\text { Würde ich unbedingt } \\
\text { empfehlen }(\%)\end{array}$ & $\begin{array}{l}\text { Würde ich bedingt } \\
\text { empfehlen }(\%)\end{array}$ & $\begin{array}{l}\text { Eher neu- } \\
\text { tral }(\%)\end{array}$ & $\begin{array}{l}\text { Würde ich eher nicht } \\
\text { empfehlen }(\%)\end{array}$ & $\begin{array}{l}\text { Davon würde ich } \\
\text { abraten }(\%)\end{array}$ & $\begin{array}{l}\text { Anzahl der } \\
\text { Bewertungen }\end{array}$ \\
\hline Diaspora & 39,39 & 30,30 & 21,21 & 6,06 & 3,03 & 33 \\
Mastodon & 45,95 & 24,32 & 24,32 & 5,41 & 0 & 37 \\
Matrix.org & 25,81 & 38,71 & 22,58 & 6,45 & & 31 \\
bzw. Riot & & & & & 0 & \\
Friendi.ca & 14,29 & 42,86 & 35,71 & 7,14 & 7,69 & 28 \\
SCION & 0 & 7,69 & 84,62 & 0 & 4,76 & 13 \\
Jami & 0 & 9,52 & 85,71 & 0 & 7,14 & 6,25 \\
Utopia & 0 & 0 & 92,86 & 0 & 0 & 14 \\
OpenBazaar & 0 & 12,5 & 81,25 & 0 & 6,25 \\
\hline
\end{tabular}

Tab. 2 Stärken und Schwächen von diaspora

\begin{tabular}{|c|c|c|}
\hline & Stärken & Schwächen \\
\hline $\begin{array}{l}\text { Benutzer- } \\
\text { freund- } \\
\text { lichkeit }\end{array}$ & $\begin{array}{l}\text { Siebenmal wurde die einfache Bedienbarkeit gelobt } \\
\text { „Diaspora gibt dem User die Kontrolle. Könnte jeder } \\
\text { selbst administrieren“ } \\
\text { „Einfache Oberfläche WENN man mal kapiert hat, wel- } \\
\text { che Philosophie dahinter steckt“ }\end{array}$ & $\begin{array}{l}\text { „Zu komplex“ hieß es einmal } \\
\text { „Man braucht erstmal einen Übersetzer, um zu verstehen, dass } \\
\text { „Aspekte ' Gruppen sind. Das Problem mit der ,eigenen Sprache“ } \\
\text { haben recht viele Netzwerke, was für viele Menschen eine Ein- } \\
\text { stiegshürde ist, die nicht gerade klein ist“ } \\
\text { „Verbesserungswürdig wäre es, wenn das Aufsetzen und Updaten } \\
\text { eigener Diaspora-Pods so einfach wäre wie z. B. bei Nextcloud.“ }\end{array}$ \\
\hline Funktionen & $\begin{array}{l}\text { „Es funktioniert und für mich fehlten keine Features“ } \\
\text { „Kommunikation in eigenen Aspekten (Kanälen)“ } \\
\text { „Voll anonym, keine Tracker, wenig Skripte“ } \\
\text { „Meine Daten sind meine Daten“ } \\
\text { „Was ich sehe, bestimme ich“ }\end{array}$ & $\begin{array}{l}\text { Sechsmal wurden fehlende Features bemängelt, z.B. das Editieren } \\
\text { von Beiträgen } \\
\text { „Schwierig, Nachrichten zu archivieren/wiederzufinden. Schwierig, } \\
\text { Nutzer nachhaltig zu blocken, die illegale Inhalte verbreiten oder } \\
\text { Nutzer belästigen. (Wie eben auch in jedem anderen dezentralen } \\
\text { Netz)“ } \\
\text { „Außerdem hat die Suche viele Schwächen - man kann z.B. nur } \\
\text { nach einem \#Tag suchen und nicht mehrere kombinieren oder aus- } \\
\text { schließen“ } \\
\text { „Accounts sind an eine Instanz gebunden und können nicht auf } \\
\text { andere übertragen werden“ }\end{array}$ \\
\hline Technologie & $\begin{array}{l}\text { Die Ausgereiftheit und Ausfallsicherheit wird gelobt, } \\
\text { dreimal die Kontrolle über die eigenen Daten }\end{array}$ & $\begin{array}{l}\text { Die Kommunikation zwischen den Pods wird zweimal bemängelt, } \\
\text { weil sie nicht gut funktioniert. } \\
\text { Dreimal wurde die fehlende Interoperabilität mit dem Fediverse und } \\
\text { mit anderen Netzen vermisst. } \\
\text { „Alles liegt unverschlüsselt auf dem Server vor, auch private Nach- } \\
\text { richten“ } \\
\text { Einmal wurde eine nicht optimale Performance kritisiert }\end{array}$ \\
\hline Hintergrund & $\begin{array}{l}\text { „Kein kommerzielles Interesse“ } \\
\text { „Open-source“" } \\
\text { „Werbefrei“ }(2 \times) \\
\text { „Zensurbeständigkeit“" }\end{array}$ & „Langsame Entwicklung“ \\
\hline Community & $\begin{array}{l}\text { „Niveau! Es werden niveauvolle Themen und Unterhal- } \\
\text { tungen gepflegt, öfter mit Recherche-Quellen-Angaben. } \\
\text { Die Admins achten darauf, dass es keinen Spam, kei- } \\
\text { ne Trolle, etc. gibt. Falls es welche schaffen, werden } \\
\text { sie rasch gelöscht oder die Community macht es ihnen } \\
\text { schwer“ } \\
\text { „Nette Nutzerbasis“ } \\
\text { "Gute Community“ (2×) } \\
\text { „Es gibt relativ viele deutsche Nutzer*innen“ } \\
\text { "Verbreitet“" } \\
\text { "Weltweit" } \\
\text { „Aktive Entwickler-Community; Benutzer sind like-min- } \\
\text { ded people“ }\end{array}$ & $\begin{array}{l}\text { „Beiträge von anderen Benutzern sind inhaltlich eher alternativ und } \\
\text { techniklastig - schreckt manche neuen Nutzer ab“ } \\
\text { „Bisher wenig registrierte Nutzende - kaum Möglichkeiten, sich } \\
\text { mit Bekannten auszutauschen“ } \\
\text { „Freunde sind nicht so leicht zu finden“ } \\
\text { „Wenig aktive Nutzer“ }(3 \times)\end{array}$ \\
\hline
\end{tabular}


Tab. 3 Stärken und Schwächen von Mastodon

\begin{tabular}{|c|c|c|}
\hline & Stärken & Schwächen \\
\hline $\begin{array}{l}\text { Benutzer- } \\
\text { freund- } \\
\text { lichkeit }\end{array}$ & $\begin{array}{l}\text { „Einfach zu nutzen, in der App wie in der Website“ }(3 \times) \\
\text { „Zweckmäßiger Dienst, nicht überladen“ } \\
\text { „Optische Ähnlichkeit mit Twitter macht die Umstellung ein- } \\
\text { fach“ } \\
\text { „Modernes User Interface“ }\end{array}$ & $\begin{array}{l}\text { „Unübersichtlich“ }(2 \times) \\
\text { „Für Anfänger*innen ungewohnte Accountnamen, bei z. B. } \\
\text { mobilen Clients werden beispielsweise nicht alle Posts zu } \\
\text { einem Hashtag angezeigt“ } \\
\text { „keine Formatierung“ } \\
\text { „Serverbetrieb ist nicht trivial“ }(2 \times)\end{array}$ \\
\hline Funktionen & $\begin{array}{l}\text { „Vollwertige Twitter-Alternative mit mehr Features“ }(3 \times) \\
\text { „Zudem sind die Algorithmen der Timeline fairer als bei Twitter } \\
\text { (nicht von Sensationen getrieben, um Leute bei der Plattform zu } \\
\text { halten)“ }\end{array}$ & - \\
\hline Technologie & $\begin{array}{l}\text { „Technisch ausgereift und stabil“ }(2 \times) \\
\text { „Plattformübergreifend unterstützt“" } \\
\text { „ActivitiPub als Protokoll zur Kommunikation zwischen ver- } \\
\text { schiedensten Plattformen und die daraus resultierende Interak- } \\
\text { tivität. Auch zwischen Kommentarspalten von Webseiten und } \\
\text { Mastodon“ }(2 \times)\end{array}$ & $\begin{array}{l}\text { „Keine Ende-zu-Ende-Verschlüsselung“ } \\
\text { „Förderierter Ansatz braucht auch zentrale Anbieter (wenn } \\
\text { nicht nur einen, sondern mehrere)“ } \\
\text { „Die Entwickler gehen gerne mal eigene Wege, die vom } \\
\text { Protokollstandard abweichen. Das ist eher schädlich als hilf- } \\
\text { reich.“ }\end{array}$ \\
\hline Hintergrund & $\begin{array}{l}\text { „Open Source“ }(2 \times) \\
\text { „Ohne kommerzielle Interessen“ }(2 \times) \\
\text { „Werbefrei“ }(3 \times)\end{array}$ & - \\
\hline Community & $\begin{array}{l}\text { „Keine Profilbildung, keine Trolls“ } \\
\text { sehr viele Nutzer*innen }(8 \times) \\
\text { „Viele Beiträge zu Themen, die mich interessieren wie freie } \\
\text { Software, Nachhaltigkeit, Softwareentwicklung, Datenschutz, } \\
\text { Politik, Demokratie“ }\end{array}$ & $\begin{array}{l}\text { Fehlt noch an Bekanntheit }(4 \times) \\
\text { „Monokultur“ } \\
\text { „Schwer zu kontrollieren (e.g. Hate Speech), Moderation nur } \\
\text { durch lokale Admins möglich“ } \\
\text { „Keine vernünftigen Diskussionen möglich“ }(2 \times)\end{array}$ \\
\hline
\end{tabular}

\section{Technologien für die asynchrone Kommunikation}

Ein dezentralisiertes soziales Netzwerk: friendi.ca Diese freie Software bietet die wichtigsten Funktionalitäten eines sozialen Netzwerks: Posts, Likes und Kommentare, Fotoalben und Veranstaltungskalender, private Diskussionsgruppen, aber auch das Löschen von Beiträgen, Herunterladen der eigenen Daten und verschlüsselte Kommunikation [4].

\section{Ein weiteres dezentralisiertes soziales Netzwerk: diaspora} Diaspora ist älter als friendi.ca und ebenfalls eine freie Software, ausdrücklich mit dem obersten Ziel entwickelt, den Datenschutz der Benutzer zu gewährleisten. Hier ist auch eine anonyme Teilnahme unter Pseudonym erlaubt. Überall auf der Welt gibt es Pods, also Server, auf denen man sich anmelden kann, ohne selbst einen Server betreiben zu müssen. 2018 hatte das Netzwerk 665.000 registrierte Benutzer*innen [5].

Dezentralisiertes Mikroblogging: Mastodon Während die beiden vorigen Netzwerke als dezentrale Facebook-Alternativen gedacht sind, ersetzt Mastodon Twitter. Hier können Sie schreiben, lesen, anderen Benutzer*innen folgen. Anfang 2019 hatten über 1,8 Mio. Nutzer*innen 202 Mio. Nachrichten von 2800 Instanzen aus versendet [6].

Mastodon und Friendica sind Teil eines größeren Netzwerks von miteinander technisch kompatiblen Technologien, dem sogenannten Fediverse [7, 8].

\section{Technologien für die synchrone Kommunikation}

Dezentralisierte Echtzeitkommunikation: Matrix.org und Riot.im Diese Open-Source-Projekte bieten eine Ende-zuEnde-verschlüsselte Echtzeitkommunikation (Chat) ohne Single Point of Failure oder zentrale Kontrolle [9, 10].

Eine freie und universelle Kommunikationsplattform für Telefon- und Videokonferenzen: Jami Jami ist ein quelloffenes und universelles Kommunikationstool, basierend auf einer verteilten Architektur. Es bietet Textnachrichten, Videound Audioanrufe, Dateiübertragung und Videokonferenzen [11].

Dezentralisiertes Computernetzwerk: Utopia In diesem Peer-to-Peer-Netzwerk können Sie nicht nur Textnachrichten senden und Diskussionschats führen, sondern auch Dateien übertragen und E-Mails senden. Die Software befindet sich gerade im Beta-Test [12].

\section{Marktplätze}

Dezentralisierter Marktplatz: OpenBazaar Auch für Ebay gibt es eine dezentralisierte Variante: Hier läuft der Handel nicht über einen Mittelsmann, sondern Käufer und Verkäufer verbinden sich direkt miteinander. Bezahlt wird in Kryptowährungen [13, 14]. 
Tab. 4 Stärken und Schwächen von Matrix.org mit Riot

\begin{tabular}{|c|c|c|}
\hline & Stärken & Schwächen \\
\hline $\begin{array}{l}\text { Benutzer- } \\
\text { freund- } \\
\text { lichkeit }\end{array}$ & $\begin{array}{l}\text { „Ansprechende Weboberfläche bzw. App“ } \\
\text { „Relativ einfach zu betreiben, gute Clients } \\
\text { (Riot)““ }\end{array}$ & „Erfordert noch technisches Verständnis“ \\
\hline Funktionen & $\begin{array}{l}\text { „Chat mit Zusatzfunktionen, z. B. Mark- } \\
\text { down“ }(2 \times) \\
\text { „Bietet alles, was ein moderner Messenger } \\
\text { braucht“ } \\
\text { „Featurereicher Messenger auf Basis dezen- } \\
\text { traler Knoten“ } \\
\text { „Zu unterschiedlicher Kommunikation nutz- } \\
\text { bar“ } \\
\text { „Bietet Widgets wie Etherpads, die extrem } \\
\text { hilfreich sind, um in Chats mit vielen Men- } \\
\text { schen Zusammenfassungen und Informatio- } \\
\text { nen stets schnell zugreifbar und aktuell zu } \\
\text { halten“ }\end{array}$ & $\begin{array}{l}\text { „Versucht, das Rad neu zu erfinden“ } \\
\text { „Noch ist die Löschung von Raum-Historien nicht möglich. Aber ist schon in } \\
\text { Arbeit (Recht auf Vergessen)“ } \\
\text { „Audio- und Videochat hat nicht funktioniert, als ich es das letzte Mal getestet } \\
\text { habe. Selbst Textchat glitcht manchmal beim Scrollen. Software ist nicht stabil } \\
\text { und nutzbar“ }\end{array}$ \\
\hline Technologie & $\begin{array}{l}\text { „Ähnelt sehr stark Slack und IRC und ist für } \\
\text { die Kommunikation in komplexeren Struktu- } \\
\text { ren mit vielen Leuten geeignet, da Inhalte auf } \\
\text { verschiedene Kanäle verteilt werden können“ } \\
\text { „Verschlüsselung“ }(3 \times) \\
\text { „Ordentliches Protokoll“ } \\
\text { Bietet Bridges/Connectoren zu vielen ande- } \\
\text { ren Netzwerken (4×) } \\
\text { „Auf Föderierbarkeit designte Namenssche- } \\
\text { mata“ } \\
\text { „API first“ } \\
\text { „Gute Weiterentwicklungen in den letzten } \\
\text { Jahren“ } \\
\text { „Moderne Architektur im Gegensatz zu } \\
\text { XMPP, dadurch sind Videokonferenzen einfa- } \\
\text { cher möglich und moderne Messenger-Featu- } \\
\text { res wie Emoticons“ } \\
\text { „Erlaubt das Aufsetzen eigener Server mit } \\
\text { moderatem Aufwand““ } \\
\text { „Dezentrale Authentifizierung“ } \\
\text { „Echtzeitkommunikation“ } \\
\text { „Funktioniert wohl gut auf diversen Geräten“ } \\
\text { „Bringt frischen Wind in Richtung IM-Lö- } \\
\text { sungen“ }\end{array}$ & $\begin{array}{l}\text { „Der Homeserver braucht sehr viel Ressourcen (Datenbank, CPU)“ }(2 \times) \\
\text { „Hat einen massiven Overhead, da das Protokoll recht kompliziert aufgebaut } \\
\text { ist und am Ende die Ressourcen mies benutzt werden (Server sind bei kleinen } \\
\text { Nutzerzahlen mitunter stark ausgelastet)“ } \\
\text { „Zudem ist die Verschlüsselung nicht standardmäßig aktiv“ } \\
\text { Verschlüsselung ist komplex und fehleranfällig }(2 \times) \\
\text { „Persistente Kommunikation, Monokultur (trotz vieler Clients)“ } \\
\text { „kein IETF-Standard (Internet). Für ein Kommunikationsprotokoll ein No-Go.“ } \\
\text { „Software ist nicht kompatibel zu xmpp oder anderen“ } \\
\text { „Clients sind in Javascript geschrieben anstatt alternativer Anwendungen. Das } \\
\text { ist ein Unding und schlimmer Trend! https://www.heise.de/security/meldung/ } \\
\text { Skype-Slack-VS-Code-Atom-Electron-Apps-haben-eine-gefaehrliche- } \\
\text { Achilles-Ferse-4493195.html“ } \\
\text { „[Verwendete Technologie, das Electron-Framework, hat Sicherheitsschwach- } \\
\text { stelle]“ } \\
\text { „Dominanz des matrix.io-Servers/Namensraums Riot ist ein tendenziell über- } \\
\text { ladener, einschüchternder Client. Absicherung verschlüsselter Räume angst- } \\
\text { einflößend; individuelle Authentifizierung aller teilnehmenden Clients (nicht } \\
\text { Personen) in den meisten Fällen unrealistisch, fehlender Fallback auf Trust- } \\
\text { Delegation (jeder Benutzer zertifiziert seine eigenen Clients, web on trust) für } \\
\text { normal vertrauliche Kommunikation. Kompromittierung von Servern und der } \\
\text { offiziellen Build-Umgebung schwächt das Vertrauen in die Lösung grundsätz- } \\
\text { lich“ }\end{array}$ \\
\hline Hintergrund & $\begin{array}{l}\text { „Open Source“ } \\
\text { „Unabhängig von Konzernen und deren Inte- } \\
\text { ressen“ } \\
\text { „europäische Stiftung“ } \\
\text { „werbefrei“ }(2 \times)\end{array}$ & $\begin{array}{l}\text { „Siehe Beschreibung auf https://matrix.org/legal/privacy-notice Außerdem wer- } \\
\text { den die privaten Schlüssel wohl auf dem Server gehalten, wenn ich es richtig } \\
\text { verstehe. [Zentrale Datenhaltung der Benutzerdaten. Es gibt technische und } \\
\text { rechtliche Grenzen beim Recht auf Vergessen, d.h. Löschen von Inhalten, insbe- } \\
\text { sondere die an Twitter weitergegeben wurden]“" } \\
\text { „Noch Abhängigkeit von vector.im“" } \\
\text { „VC-finanziert (VC = Venture Capital)“ }\end{array}$ \\
\hline Community & „Community“ & „Zu wenig bekannt“ $(3 \times)$ \\
\hline
\end{tabular}

\section{Weitere dezentrale Technologien}

Die beiden folgenden Technologien wollen das Internet neu erfinden.

dfinity ist eine serverlose Infrastruktur, eine manipulationssichere offene Cloud-Plattform mit ICP-Protokoll, das eine Blockchain bildet. Die teilnehmenden Datenzentren müssen sich registrieren. Skalierbarkeit, Datenschutz und Sicherheit sind gleichzeitig das Ziel, aber auch die Unab- hängigkeit von den Produkten der großen Softwarefirmen [15].

SCION verspricht eine sichere Internetarchitektur als Open Source, die vor allem für Sicherheit und Verfügbarkeit entwickelt wurde. Dazu gehört Transparenz über den Pfad von Ende zu Ende und die Auswahl sicherer Pfade (Pfadkontrolle) durch den Benutzer, starke Resilienz durch eine skalierbare Routing-Infrastruktur sowie ausdrückliche Trust-Informationen. In zehn Jahren wurde das Internet neu erfunden [16, 17]. 
Tab. 5 Stärken und Schwächen von friendi.ca

\begin{tabular}{|c|c|c|}
\hline & Stärken & Schwächen \\
\hline $\begin{array}{l}\text { Benutzer- } \\
\text { freund- } \\
\text { lichkeit }\end{array}$ & „Einfach einzurichten“ & $\begin{array}{l}\text { „Sieht etwas altbacken aus und fühlt sich } \\
\text { auch so an“ } \\
\text { „Das Standard-Theme könnte etwas } \\
\text { moderner sein“ } \\
\text { „Unbequemes Handling, nur für tech- } \\
\text { nisch sehr versierte Menschen nutzbar“ } \\
\text { „Sehr umständlich, kaum brauchbare } \\
\text { Informationen, keine Tutorials. Es ist } \\
\text { unmöglich, in kurzer Zeit befriedigende } \\
\text { Ergebnisse zu erhalten. Hohes Frustrati- } \\
\text { onspotenzial“ }\end{array}$ \\
\hline Funktionen & $\begin{array}{l}\text { „L̈̈schung von geschriebenen Nachrichten“ } \\
\text { „Man kann sehr gut selbst differenzieren, was man sehen will und was nicht, und } \\
\text { auch wer welche Posts von einem zu sehen bekommt. In der Hinsicht ist friendica } \\
\text { wahrscheinlich allen anderen sozialen Netzwerken überlegen. Außerdem kann man } \\
\text { von dort mit verschiedenen Protokollen kommunizieren (z. B. Diaspora, Mastodon). } \\
\text { Kommunikativ (viele Protokolle), vielfältig (Blogs, Foren, Microblogs, private Kom- } \\
\text { munikation) Umfangreich konfigurierbar“ } \\
\text { „Diskussionen werden übersichtlich und gut verfolgbar dargestellt - Hoher Fokus } \\
\text { auf Konnektivität mit anderen Netzwerken - Hoher Fokus auf Privatsphäre - Zeit- } \\
\text { basiertes Löschsystem ist integriert (Recht auf Vergessen) - Foren, die mit anderen } \\
\text { Friendica-Knoten föderiert werden - Events/Kalender (föderiert)“ }\end{array}$ & $\begin{array}{l}\text { „Sehr großer Funktionsumfang, eher } \\
\text { unübersichtlich“ }\end{array}$ \\
\hline Technologie & $\begin{array}{l}\text { „DFRN Protokoll““ } \\
\text { „Ausfallsicher“ }\end{array}$ & $\begin{array}{l}\text { „Wenig Protokolle“" } \\
\text { „Manchmal langsam“ }\end{array}$ \\
\hline Hintergrund & $\begin{array}{l}\text { „Open-source“" } \\
\text { „Werbefrei““ }\end{array}$ & - \\
\hline Community & „Community“ & „Eher nicht bekannt bzw. verbreitet“ \\
\hline
\end{tabular}

Tab. 6 Stärken und Schwächen von Jami

\begin{tabular}{lll}
\hline & Stärken & Schwächen \\
\hline $\begin{array}{l}\text { Benutzerfreundlich- } \\
\text { keit }\end{array}$ & $\begin{array}{l}\text { „Gute Benutzungs- } \\
\text { oberfläche, einfache }\end{array}$ & - \\
Bedienung“ & \\
Funktionen & - & - \\
Technologie & „Gutes Privacy by & „Bedarf noch Entwicklung. Läuft nicht immer stabil, kann aber auch an schlechter Internet- \\
& Design“ & ,Situng in D liegen“ \\
& & „Ist noch nicht fertig entwickelt und einsatzbereit“ \\
& & „Ist auch nicht mit anderer Software kompatibel wie XMPP oder Matrix““ \\
Hintergrund & - & ,Mangelnde Verbreitung“ \\
Community & - &
\end{tabular}

Tab. 7 Stärken und Schwächen von OpenBazaar

\begin{tabular}{|c|c|c|}
\hline & Stärken & Schwächen \\
\hline $\begin{array}{l}\text { Benutzerfreund- } \\
\text { lichkeit }\end{array}$ & „Ich hab's nur oberflächlich getestet und es hat alles funktioniert.“ & - \\
\hline Funktionen & $\begin{array}{l}\text { „Ist mir vor allem als dezentraler Marktplatz ein Begriff und in die- } \\
\text { ser Hinsicht nahezu alternativlos.“ }\end{array}$ & „Es hat sicher auch noch einige Probleme ...“ \\
\hline Technologie & - & - \\
\hline Hintergrund & - & - \\
\hline Community & - & $\begin{array}{l}\text { „Eventuell könnte man sagen, dass es recht neu } \\
\text { ist und wenig Nutzer hat““ }\end{array}$ \\
\hline
\end{tabular}




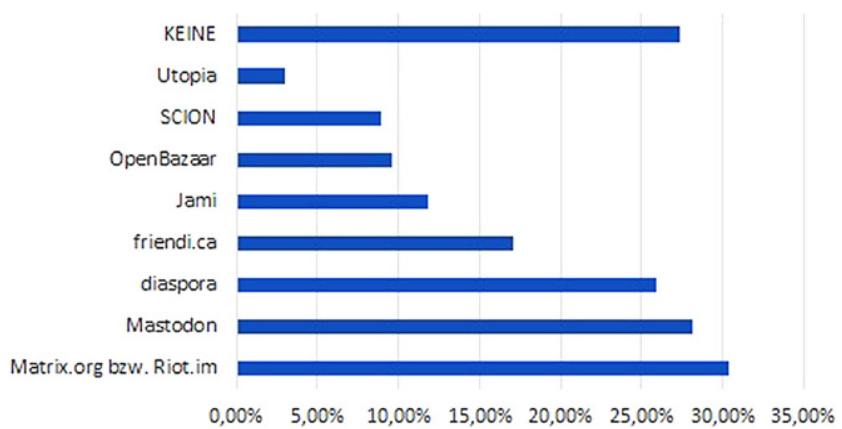

Abb. 1 Welche der Technologien würden Sie gerne benutzen?

Straightway ist ein Open-Source Peer-to-Peer Netzwerk, das ohne zentrale Anbieter auskommt, Inhalte verschlüsselt und Metadaten verbirgt. Daten können anonym gespeichert und geteilt werden. Darauf aufbauend erlaubt Straightway die Entwicklung von Kommunikationsanwendungen wie z. B. sozialen Netzwerken, die Datenschutzaspekte auf technischer Ebene im Sinne von Privacy by Design konsequent angehen. Die Software ist in einem frühen Entwicklungsstadium. Hilfe und Mitarbeit sind willkommen [18].

\section{Umfrageergebnisse: Bekanntheit der Technologien}

In der Umfrage wurde eine Auswahl an Technologien kurz vorgestellt und dann nach deren Bekanntheit und Erfahrungen damit gefragt. Zur Auswahl standen Friendi.ca, diaspora, Jami, Mastodon, Matrix, OpenBazaar, Riot, SCION, Utopia.

Die beiden Fragen bezüglich Bekanntheit lauteten: „Welche der genannten Technologien kennen Sie?" und „Welche der hier genannten Technologien nutzen Sie bereits?“

Zwei Technologien erreichten einen Bekanntheitsgrad von beinahe 50\%: diaspora und Mastodon (48\% respektive $45 \%)$. Zwei weitere wurden von einem Drittel der Teilnehmer*innen genannt: Matrix.org und friendi.ca (34\% respektive $29 \%$ ). Genutzt werden sie etwas weniger: diaspora und Mastodon kommen jeweils auf 28\% Nutzung, matix.org auf $24 \%$, friendi.ca auf $7 \%$ und Jami auf $4 \%$, OpenBazaar 2\%. Etwas mehr als ein Drittel der Teilnehmer/innen (38\%) kannten keine der genannten Technologien, $42 \%$ nutzen keine davon.

\section{Umfrageergebnisse: Bewertung der Technologien}

Die Umfrageteilnehmer*innen bewerteten die Technologien, die sie kennen, auf einer Likert-Skala und konnten

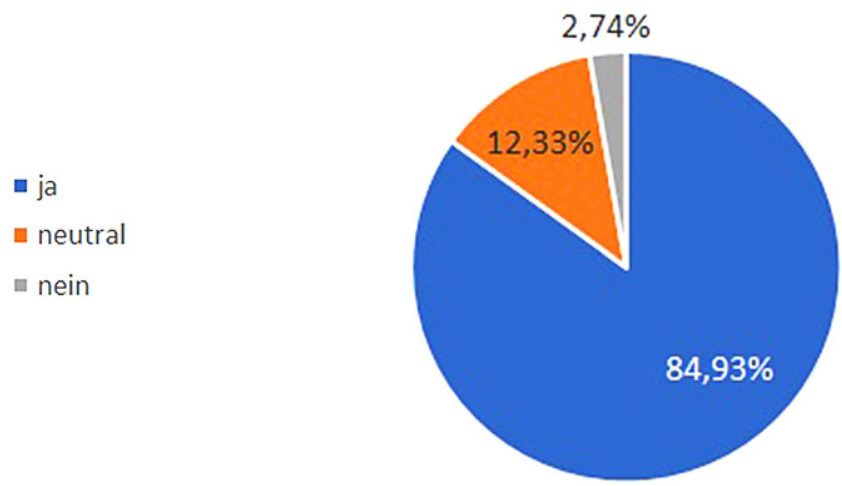

Abb. 2 Würden Sie der GI empfehlen, das Thema weiter zu verfolgen?

in Freitextformat deren Stärken und Schwächen nennen. Tab. 1 zeigt, wie viele Befragte welche Technologie empfehlen. Die beste Bewertung erhielten diaspora, Mastodon und Matrix.org.

Die folgenden Tab. 2, 3, 4, 5, 6 und 7 listen die Stärken und Schwächen der Technologien sortiert nach folgenden fünf Kategorien auf: Benutzerfreundlichkeit, Funktionen, Technologie, Hintergrund, Community. Sie sind die Antworten auf die Fragen „Welche Stärken hat diese Lösung?“ und „Welche Schwächen hat diese Lösung?“ Diese Antworten sind natürlich subjektiv und müssen nicht unbedingt so stimmen.

Für SCION und Utopia gab es keine Kommentare.

\section{Umfrageergebnisse: Welche Technologie würden Sie gerne benutzen?}

Anschließend wurden die Teilnehmer*innen noch gefragt: „Nach allem, was Sie über die genannten Technologien wissen (oder davon gehört haben): Welche davon würden Sie gerne benutzen?" Abb. 1 zeigt die Auswertung der insgesamt 135 Antworten auf diese Frage. Auch hier hatten wieder dieselben drei Angebote die Nase vorne, während $27 \%$ der Befragten keine ausprobieren wollten.

\section{Umfrageergebnisse: Weitere Technologien?}

Nach weiteren Technologien befragt, nannten die Befragten zahlreiche Namen. Am weitaus häufigsten genannt wurden Hubzilla, XMPP und Conversations.

Hubzilla ist ein dezentrales soziales Netzwerk [19, 20]. $\mathrm{xmpp} / \mathrm{Jabber}$ ist ein offener Standard für Messenger [21, 22].

Conversations basiert auf XMPP und ist ein Messenger für Android [23, 24].

Tab. 8 listet weitere Technologien auf. Die meisten davon waren Einmalnennungen. 
Tab. 8 Weitere dezentrale Technologien

\begin{tabular}{|c|c|c|}
\hline Name & Kurze Beschreibung & URLs \\
\hline Activity Pub & Dezentralisiertes Protokoll für soziale Netzwerke & https://activitypub.rocks/ https://www.w3.org/TR/activitypub/ \\
\hline Bitcoin & Zahlungsnetzwerk & https://bitcoin.org/de/ \\
\hline BitTorrent & P2P Filesharing und Streaming & https://www.bittorrent.com/lang/de/ \\
\hline Briar & Instant Messaging & https://briarproject.org/ \\
\hline DeltaChat & Chatten über E-Mail & https://delta.chat/de/ \\
\hline Etherpad & $\begin{array}{l}\text { Online-Texteditor zur kollaborativen Erstellung von Tex- } \\
\text { ten }\end{array}$ & https://etherpad.org/ \\
\hline Funkwhale & Eine Community zum Austausch von Musik & https://funkwhale.audio/ \\
\hline GNUnet & Neues Internetprotokoll & https://gnunet.org \\
\hline Gnu-social & $\begin{array}{l}\text { Standard zur Kommunikation zwischen Microblogging- } \\
\text { Diensten }\end{array}$ & https://www.gnu.org/software/social/ \\
\hline Gnutella & P2P Filesharing & $\begin{array}{l}\text { https://sourceforge.net/projects/gtk-gnutella/ } \\
\text { http://www.gnutellaforums.com/ }\end{array}$ \\
\hline $\begin{array}{l}\text { Internet Relay } \\
\text { Chat IRC }\end{array}$ & Textbasiertes Chatsystem & https://de.wikipedia.org/wiki/Internet_Relay_Chat \\
\hline IPFS & Protokoll für verteilte Netzwerke & https://ipfs.io/ \\
\hline Jitsi & Videokonferenzen & https://jitsi.org/ \\
\hline Libertree & $\begin{array}{l}\text { Software, mit der man ein soziales Netzwerk entwickeln } \\
\text { kann }\end{array}$ & http://libertree.org/ \\
\hline Mattermost & Messaging-Plattform & https://mattermost.com/ \\
\hline Mobilizon & Soziales Netzwerk & https://joinmobilizon.org/ \\
\hline $\begin{array}{l}\text { Mozilla Webt- } \\
\text { hings }\end{array}$ & Dezentralisiertes Internet of Things & https://iot.mozilla.org/about/ \\
\hline Mumble & Telefonkonferenzen & https://www.mumble.com/ \\
\hline Namecoin & Dezentrale DNS-Alternative, Identitätsmanagement & https://www.namecoin.org/ \\
\hline Nextcloud & $\begin{array}{l}\text { Private Cloud für die Installation auf eigenem Rechner, } \\
\text { kommerziell }\end{array}$ & https://nextcloud.com/ \\
\hline Okuna Social & Soziales Netzwerk & $\begin{array}{l}\text { https://medium.com/okuna } \\
\text { https://github.com/OkunaOrg/okuna-app }\end{array}$ \\
\hline OnionShare & P2P Filesharing & https://onionshare.org/ \\
\hline Owncloud & Open-Source-Cloud & https://owncloud.org/ \\
\hline PeerTube & Dezentralisierte Videoplattform & $\begin{array}{l}\text { https://joinpeertube.org/ } \\
\text { https://joinpeertube.org/de/ } \\
\text { https://peertube.live/ } \\
\text { https://peertube.cpy.re/ }\end{array}$ \\
\hline Pixelfed & Teilen von Fotos & https://pixelfed.org/ \\
\hline Pleroma & $\begin{array}{l}\text { Serversoftware für soziale Netzwerke mit offenen Proto- } \\
\text { kollen }\end{array}$ & $\begin{array}{l}\text { https://pleroma.social/ } \\
\text { https://www.pleroma.com/main/all }\end{array}$ \\
\hline Plume & Blogging & https://joinplu.me/ \\
\hline Quicksy & Ein Spin-off des XMPP-Messengers Conversations & $\begin{array}{l}\text { https://quicksy.im/ } \\
\text { https://www.hse-it.de/apps-android/quicksy } \\
\text { https://www.freie-messenger.de/sys_xmpp/conversations/\# } \\
\text { quicksy }\end{array}$ \\
\hline Reclaim:ID & Dezentrale Verwaltung von digitalen Identitäten & $\begin{array}{l}\text { https://reclaimid.gitlab.io/ } \\
\text { https://gitlab.com/reclaimid }\end{array}$ \\
\hline RocketChat & Chat-Software & $\begin{array}{l}\text { https://rocket.chat/ } \\
\text { https://github.com/RocketChat/Rocket.Chat }\end{array}$ \\
\hline Sandstorm.io & Selbsthosting von Web-Apps & Sandstorm.io \\
\hline Scuttlebutt & Soziales Netzwerk & https://scuttlebutt.nz/ \\
\hline Searx.me & Meta-Suchmaschine & $\begin{array}{l}\text { https://www.searx.me/ } \\
\text { https://en.wikipedia.org/wiki/Searx }\end{array}$ \\
\hline Signal & Ein verschlüsselter Text-, Sprach- und Videomessenger & $\begin{array}{l}\text { https://www.signal.org/de/ } \\
\text { https://de.wikipedia.org/wiki/Signal_(Messenger) }\end{array}$ \\
\hline
\end{tabular}


Tab. 8 (Fortsetzung)

\begin{tabular}{|c|c|c|}
\hline Name & Kurze Beschreibung & URLs \\
\hline Solid & $\begin{array}{l}\text { Eine neue Architektur für datenschutzkonforme Anwen- } \\
\text { dungen }\end{array}$ & https://solid.inrupt.com/ \\
\hline Taler & Ein Online-Bezahlsystem & https://taler.net/ \\
\hline Telegram & Messenger & $\begin{array}{l}\text { https://telegram.org/ } \\
\text { https://telegram.de/ }\end{array}$ \\
\hline Threema & Messenger & https://threema.ch/de/ \\
\hline Tor & Anonymes Surfen im Internet & $\begin{array}{l}\text { https://www.torproject.org/de/ } \\
\text { https://de.wikipedia.org/wiki/Tor_\%28Netzwerk\%29 }\end{array}$ \\
\hline Urbit & $\begin{array}{l}\text { Urbit OS ist ein Betriebssystem, das auf Linux als ,over- } \\
\text { lay OS“ läuft, Urbit ID ist ein Identitätsmanagementsys- } \\
\text { tem }\end{array}$ & Urbit.org \\
\hline Write freely & Blogging & https://writefreely.org/ \\
\hline YaCy & Dezentralisierte P2P-Suchmaschine & $\begin{array}{l}\text { https://www.yacy.net/ } \\
\text { https://de.wikipedia.org/wiki/YaCy }\end{array}$ \\
\hline
\end{tabular}

\section{Fazit und Ausblick}

Wie geht es weiter mit diesem Thema? Abb. 2 zeigt die Verteilung der 146 Antworten auf die Frage „Würden Sie der GI [Gesellschaft für Informatik] empfehlen, das Thema weiter zu verfolgen?“ Das Ergebnis ist also eindeutig ,ja“.

Zuletzt bekräftigten die Teilnehmer in ihren Freitextkommentaren die Bedeutung des Themas. Einer schrieb: „Das Thema empfinde ich als wichtig. Wenn es weiterverfolgt werden sollte, wäre es meiner Meinung nach wichtiger, es in die Gesellschaft zu tragen als innerhalb der GI zu diskutieren, da meiner Erfahrung nach Informatiker und Informatikerinnen eh schon eine Affinität zum Thema haben. Allerdings sollte diese Einschätzung auch überprüft werden."

Das ist doch eine gute Idee für die nächsten Aktivitäten. Als Informatiker*innen gestalten wir die Zukunft der Digitalisierung. Hoffentlich in die richtige Richtung!

Funding Open Access funding provided by Projekt DEAL.

Open Access Dieser Artikel wird unter der Creative Commons Namensnennung 4.0 International Lizenz veröffentlicht, welche die Nutzung, Vervielfältigung, Bearbeitung, Verbreitung und Wiedergabe in jeglichem Medium und Format erlaubt, sofern Sie den/die ursprünglichen Autor(en) und die Quelle ordnungsgemäß nennen, einen Link zur Creative Commons Lizenz beifügen und angeben, ob Änderungen vorgenommen wurden.

Die in diesem Artikel enthaltenen Bilder und sonstiges Drittmaterial unterliegen ebenfalls der genannten Creative Commons Lizenz, sofern sich aus der Abbildungslegende nichts anderes ergibt. Sofern das betreffende Material nicht unter der genannten Creative Commons Lizenz steht und die betreffende Handlung nicht nach gesetzlichen Vorschriften erlaubt ist, ist für die oben aufgeführten Weiterverwendungen des Materials die Einwilligung des jeweiligen Rechteinhabers einzuholen.
Weitere Details zur Lizenz entnehmen Sie bitte der Lizenzinformation auf http://creativecommons.org/licenses/by/4.0/deed.de.

\section{Literatur}

1. https://larrysanger.org/2019/06/declaration-of-digital-independence/. Zugegriffen: 23.03.2020

2. https://webisours.github.io/. Zugegriffen: 23.03.2020

3. https://indieweb.org/. Zugegriffen: 23.03.2020

4. https://friendi.ca/, https://friendica-wiki.de/doku.php. Zugegriffen: 23.03.2020

5. https://diasporafoundation.org/. Zugegriffen: 23.03.2020

6. https://mastodon.social. Zugegriffen: 23.03.2020

7. https://fediverse.party/. Zugegriffen: 23.03.2020

8. https://the-federation.info/. Zugegriffen: 23.03.2020

9. https://matrix.org/. Zugegriffen: 23.03.2020

10. https://about.riot.im/. Zugegriffen: 23.03.2020

11. https://jami.net/. Zugegriffen: 23.03.2020

12. https://beta.u.is/. Zugegriffen: 23.03.2020

13. https://openbazaar.org/. Zugegriffen: 23.03.2020

14. https://openbazaar.com/. Zugegriffen: 23.03.2020

15. Dfinity.org. Zugegriffen: 23.03.2020

16. https://www.scion-architecture.net/. Zugegriffen: 23.03.2020

17. https://pszal.github.io/papers/SCION-book.pdf. Zugegriffen: 23.03.2020

18. https://straightway.github.io/. Zugegriffen: 23.03.2020

19. https://zotlabs.org/page/hubzilla/hubzilla-project. Zugegriffen: 23.03.2020

20. https://hub.libranet.de/page/hucope/hubzilla_pitch_lvee-2017_ theses_de. Zugegriffen: 23.03.2020

21. https://xmpp.org/. Zugegriffen: 23.03.2020

22. https://de.wikipedia.org/wiki/Extensible_Messaging_and_Presence_ Protocol. Zugegriffen: 23.03.2020

23. https://www.freie-messenger.de/sys_xmpp/conversations/. Zugegriffen: 23.03 .2020

24. https://de.wikipedia.org/wiki/Conversations_(Instant_Messenger). Zugegriffen: 23.03.2020 